\title{
The Difficulty of Establishing Effective Treatments for Lateral Elbow Tendinopathy
}

\author{
Stasinopoulos Dimitrios* \\ Chairperson/Associate Professor, Physiotherapy, Director of Cyprus Musculoskeletal and Sports Trauma Research Centre (CYMUSTR EC), Department of \\ Health Sciences, School of Sciences, Cyprus
}

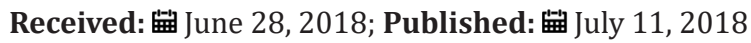

*Corresponding author: Stasinopoulos Dimitrios, Chairperson/Associate Professor, Physiotherapy, Coordinator of MSc in Sports Physiotherapy, Coordinator of Physiotherapy Program, Director of Cyprus Musculoskeletal and Sports Trauma Research Centre (CYMUSTR EC), Physiotherapy Program, Deparment of Health Sciences, School of Sciences, Nicosia, Cyprus

Abstract

Although the signs and symptoms of Lateral Elbow Tendinopathy (LET) are clear and its diagnosis is easy, to date, no ideal treatment has emerged. It is important to understand why it has proved difficult for previous workers to establish effective treatments for LET. This may be due to the difficulty of establishing nomenclature, pathophysiology, etiology, epidemiology, diagnosis and conservative treatment of the condition.

\section{Difficulties with Nomenclature}

A cursory search through existing literature reveals a plethora of terms that have been used to describe LET. These include (i) tennis elbow (TE), (ii) extensor tendonitis, (iii) extensor tendinosis, (iv) extensor tendinopathy, (v) lateral epicondylalgia, (vi) lateral epicondylitis and (vii) LET. However, it was the description of hypothetical condition by Morris of "lawn tennis arm" in 1882 that instigated the use of the term LET in medicine. LET seems to be the most appropriate term to use in clinical practice because all the other terms such as lateral epicondylitis, lateral epicondylalgia, lateral epicondylosis and/or tennis elbow make reference to inappropriate aetiological, anatomical and pathophysiological terms [1].

\section{Difficulties with Pathophysiology}

Considerable confusion concerning the actual location of LET has existed since the introduction of the term about a century and a half ago. The exact location of the pathophysiological changes was unknown for decades, since many structures around the elbow- the tendons of the wrist extensor muscles and possibly the anconeous muscle, the bursae, the radial collateral and annual ligament, the radiohumeral synovial fringe, the radiohumeral joint, the radial head and radial nerve - have been described in the pathogenesis of LET based on theoretical hypotheses and mechanisms through clinical examination and diagnosis [2]. This problem seems now to have been resolved, because the structure most commonly reported as being responsible for LET is the origin of the extensor carpi radialis brevis (ECRB) tendon on the basis of surgical findings [1].

There was also some debate as to the pathogenesis of LET. At first LET was classified as an inflammatory process and physicians attributed the pain of LET to inflammation. However, great progress has been made in this area, especially in the last two decades, and it has been found that the LET condition is a degenerative process that occurs when the ECRB has failed to heal properly after an injury or after repetitive microtrauma resulting from overuse [3]. This finding was confirmed by histopathological investigations of biopsied materials. These investigations (have resulted in a host of new findings [4]:

a) The tendon is dull, gray and friable.

b) The total amount of collagen is decreased, since breakdown exceeds repair.

c) The amounts of proteoglycans and glycosaminoglycans are increased, possibly in response to increased compressive forces associated with the repetitive motion.

d) The ratio of Type III to Type I collagen is abnormally high.

e) The normal parallel bundled fiber structure is disturbed; the continuity of the collagen is lost with disorganized fiber structure and evidence of both collagen repair and collagen degeneration.

f) Microtears, mucoid and hyaline degeneration, calcification and collagen fiber separations are seen. Many of the collagen fibers are thin, fragile, and separated from each other.

g) The number of fibroblast cells is increased; the tenocytes look different, with a more blast-like morphology (the cells look thicker, less linear). These differences show that the cells are actively trying to repair the tissue. 
h) The vascularity is increased, evidence of an immature repair process.

i) Inflammatory cells are usually not seen in the tendon.

j) Electronic microscopic observations have shown alterations in the size and shape of mitochondria in the nuclei of the tenocytes.

Based on these findings, physicians have begun to develop new or alternative theories about the source of pain associated with LET. Physicians believe that the pain of LET probably comes partly from the physical injury itself (separation of collagen fibers and mechanical disruption of tissue) and partly from irritating non-inflammatory biochemical substances that are produced as part of the injury process. The biochemical substances probably irritate the pain receptors in the tendon and surrounding area. The mechanism of pain associated with LET is yet to be confirmed by researchers and is beyond the scope of the present article.

\section{Difficulties with Etiology}

It is commonly accepted that LET is the effect of overuse, over-stress or over-exertion of the extensor tendons of the wrist, especially ECRB, by quick, continuously monotonous, repetitive and/or strenuous activities of the wrist [5]. If this were the only cause of LET, it would beg the question: why do researchers not face this cause and establish an effective treatment for LET? The answer is simple: the etiology of LET remains relatively unknown and unexplored because LET is a multifactorial condition in nature and, although the overuse of the wrist is the main cause, it is not the only one. Other factors that play a significant role in the etiology of LET can be age, sex, poor vascular supply of ECRB, anatomical variation such as differences in alignment and range of motion, decreased flexibility and cervical spine dysfunction. Even though some studies showed that LET occurs most commonly in those between 30 and 60 years of age $[3,6]$ and occurs with equal frequency in both sexes but is more severe in women $[3,6]$, no studies were found to show a relation between LET and poor vascular supply of ECRB, anatomical variation, or decreased flexibility. It has been purported that cervical spine dysfunction may contribute to the etiology of LET [5] but cervical spine dysfunction and LET are two different conditions while the symptoms of cervical spine dysfunction may mimic LET pain. Overall, a determination of the causes of LET requires further research since, if clinicians were to understand the etiology of LET, firstly an effective treatment for LE might be more easily established and secondly LET might be more easily prevented.

\section{Difficulties with Epidemiology}

The epidemiology of LET is the aspect of this condition that has been investigated in most detail, and it is clear that LET is a common clinical problem. It is generally accepted that the occurrence of LET is expressed as either an incidence or a prevalence rate [5]. The incidence rate of LET (the rate at which new cases appear over a year) is approximately 4-7 per 1000 patients per year in general practice. The annual prevalence (the number of existing cases at a given time) of this condition is $1-3 \%$ in the general population. Tennis players have been reported to account for $5-8 \%$ of all LET patients, and between $40-50 \%$ of all tennis players will be afflicted with this condition at some time during their career.

Factors such as age, gender, stress loads on the elbow and the interaction between these factors have been postulated to influence the incidence and prevalence rates of LET. Although LET occurs at all ages, the peak prevalence of LET is between 30 and 60 years (see previous section) because these are the most productive (creative) ages. The proportion of those afflicted by LET is not influenced by the sex of the patient, but the disorder appears to be of longer duration and severity in females [4] because females are weaker than males in physical characteristics such as strength. Finally, LET is almost invariably experienced in the dominant arm $(3,6)$ because this is the arm that is mainly used and is under stress in everyday activities.

If research in this area is to help clinicians establish an effective treatment for LET, it must be sustained and in depth. Future surveys of the occurrence rates of LET should carefully attend to methodological issues such as differences in sampled populations, the classification of included and excluded cases, and the validation of such cases by trained health care personnel.

\section{Difficulties with Diagnosis}

Although the diagnosis of LET is simple with the clinical picture fairly uniform, many conditions mimic LET pain, and thus the physicians can be easily misdiagnosed as LET, which complicates the prospect of optimal treatment for LET. These conditions include osteochondritis dissecans, cubital osteoarthritis, radialtunnel syndrome, rheumatoid arthritis, severe cervical spondylosis or cervical radicular syndrome, painful shoulder or rotator cuff tendinopathy and increased neural tension. However, an experienced clinician with LET patients can easily distinguish the pain of LET from the pain of other conditions that mimic LET pain.

A cursory survey of the existing literature reveals a plethora of diagnostic tests that have been used to diagnose LET $[3,6]$. These include (i) palpation on the facet of lateral epicondyle, where the ECRB tendon originates, (ii) the Tomsen test, (iii) resisted middle finger extension, (iv) the Mill's test, (v) the handgrip dynamometer test, (vi) resistance supination with the elbow in flexion and in extension, (vii) the chair test, and (viii) the coffee-cup test.

Although any therapist conducting one or more of these tests can reproduce the pain of LET, such a plethora of diagnostic tests suggests that the most variable and valid test for LET is not known. However, clinicians do not use all these tests to diagnose LET. They would normally palpitate the facet of the lateral epicondyle and one or two of the tests listed above, with tests (ii) to (v) being the most commonly used [6]. Future research might investigate these various and possibly inconsistent diagnostic tests, since different approaches to diagnosis of LET may lead to different choices of treatment for LET.

In the vast majority of LET patients the diagnosis is based on history and physical examination. Radiological investigation such as magnetic resonance imaging (MRI) or ultrasound examinations can add information in diagnosis, for example if the ECRB is the only affected structure or other structures such as supinator or 
extensor digitorum communis are also involved. Although such as investigation can help clinicians to modify their treatment in order to obtain the best therapeutic effects, it is not routinely obtained.

\section{Difficulty with Conservative Treatments}

Clinicians regarded LET as an inflammatory condition and recommended management with anti-inflammatory treatments such as NSAIDs and corticosteroid injections. However, it is now known that the LE condition is not an inflammatory process but a degenerative one (see section Difficulties with pathophysiology) and clinicians must ask themselves how efficacious treatments using these medicinal conservative approaches actually can be.

Systematic reviews of the literature failed to turn up evidence not convincingly supporting the long-term effectiveness of injections [7]. Definite conclusions cannot be drawn due to the lack of high quality studies. A systematic review found some support for the use of NSAIDs to relieve LET pain at least in the short term; however, there was, insufficient evidence either to recommend or to discourage the use of NSAIDs [8].

Some narrative reviews report cases, although they do not provide details of the nature of the cases, in which NSAIDs and corticosteroid injections provide short-term but rapid symptom relief. It is believed that NSAIDs and corticosteroid injections are effective treatments in patients with a short duration (less than six weeks) of LET. Clinicians should accept that, at least until data appear demonstrating otherwise, that these two kinds of treatments do not provide significant long-term benefit in tendinopathy such as LET. Given the known deleterious effect of corticosteroid injections into tendon and their inhibition of collagen repair when administered in the area of tendons, this treatment has lost favor. Moreover, the use of NSAIDs can cause gastrointestinal problems that impede the healing process. Therefore, the clinical use and effectiveness of these two treatments for LET are controversial. There remains a need for more effective, yet conservative and less hazardous, treatments.

Many clinicians advocate a conservative approach as the treatment of choice for LET. Physiotherapy is a conservative treatment that is usually recommended for LET patients. A wide array of physiotherapy treatments has been recommended for the management of LET. These treatments have different theoretical mechanisms of action, but all have the same aim, to reduce pain and improve function. Such a variety of treatment options suggests that the optimal treatment strategy is not known, and more research is needed to discover the most effective treatment in patients with LET.

One of the most common physiotherapy treatments for LET is an exercise programme. One consisting of eccentric exercises has shown good clinical results in LET [9] as well as in conditions similar to LET in clinical behaviour and histopathological appearance, such as patellar and Achilles tendinopathy [10]. Such an exercise programme is used as the first treatment option for our patients with LET $[11,12]$.

Malliaras and his colleagues (2013) concluded that clinicians should consider eccentric-concentric loading alongside or instead of eccentric loading in tendinopathy. Recently, isometric exercises are indicated to reduce and manage tendon pain [13-15]. LET is often related to forceful grip activities requiring isometric contraction of the wrist flexors and extensors [16]. Perhaps isometric contractions would be more beneficial than eccentric ones in lateral epicondylitis [16]. A recently clinical trial showed that the combination of concentric - eccentric training with isometric contraction had positive effects in LET [17].

\section{References}

1. Stasinopoulos D, Johnson MI (2006) 'Lateral elbow tendinopathy' is the most appropriate diagnostic term for the condition commonly referredto as lateral epicondylitis. Med Hypotheses 67(6): 1400-1402.

2. Cyriax J (1936) The pathology and treatment of tennis elbow. Journal of Bone and Joint Surgery 18: 921-935.

3. Coombes BK, Bisset L, Vicenzino B (2015) Management of lateral elbow tendinopathy: one size does not fit all. J Orthop Sports Phys Ther 45: 938-949.

4. Kraushaar B, Nirschl R (1999) Current concepts review-tendinosis of the elbow (tennis elbow). Clinical features and findings of histological immunohistochemical and electron microscopy studies*. J Bone Joint Surg Am 81(2): 259-285.

5. Vicenzino B, Wright A (1996) Lateral epicondylalgia I: Epidemiology, pathophysiology, aetiology and natural history. Physical Therapy Reviews 1(1): 23-34.

6. Bisset LM, Vicenzino B (2015) Physiotherapy management of lateral epicondylalgia. J Physiother 61: 174-181.

7. Smidt N, Assendelft WJ, Van der Windt DA, Hay EM, Buchbinder R, et al. (2002a) Corticoste-roid injections for lateral epicondylitis: A systematic review. Pain 96(1-2): 23-40.

8. Green S, Buchbinder R, Barnsley L, Hall S, White M, et al. (2002a) Nonsteroidal anti-inflammatory drugs (NSAIDs) for treating lateral elbow pain. Cochrane Database Systematic Review 2: CD003686.

9. Raman J, MacDermid JC, Grewal R (2012) Effectiveness of different methods of resistance exercises in lateral epicondylosisda systematic review. J Hand Ther 25(1): 5-25.

10. Malliaras P, Barton C, Reeves N, Langberg H (2013) Achilles and patellar tendinopathy loading programmes: a systematic review comparing clinical outcomes and identifying potential mechanisms for effectiveness. Sports Med 43(4): 267-286

11. Stasinopoulos D, Johnson MI (2004) Treatment/management for tendinopathy. The BMJ 324: 626.

12. Stasinopoulos D, Stasinopoulos I, Stasinopoulou K (2014) A pilot trial to study the effectiveness of an exercise programme in the treatment of rotator cuff tendinopathy. J Biol Exerc 10(2): 69-77.

13. Rio E, Kidgell D, Purdam C (2015) Isometric exercise induces analgesia and reduces inhibition in patellar tendinopathy. Br J Sports Med 49(19): 1277-1283.

14. Stasinopoulos D (2015) The effectiveness of isometric contractions combined with eccentric contractions and stretching exercises on pain and disability in lateral elbow tendinopathy: A case reports. J Nov Physiother 5: 1-5.

15. Young Park J, Park H, Choi J (2010) Prospective evaluation of the effectiveness of a home-based program of isometric strengthening exercises: A 12 month follow up. Clin Orthop Surg 2(3): 173-178.

16. Martinez-Silvestrini JA, Newcomer KL, Gay RE, Schaefer MP, Kortebein P, et al. (2005) Chronic lateral epicondylitis: comparative effectiveness of a home exercise program including stretching alone versus stretching supplemented with eccentric or concentric strengthening. J Hand Ther 18(4): 411-419. 
17. Stasinopoulos D, Stasinopoulos I (2017) Comparison of effects of eccentric training, eccentric - concentric training and eccentric concentric training combined with isometric contraction in the treatment of Lateral Elbow Tendinopathy. Journal of hand therapy 30(1): 13-19.

ISSN: 2574-1241

DOI: 10.26717 /BJSTR.2018.06.001390

Stasinopoulos Dimitrios. Biomed J Sci \& Tech Res

(C) This work is licensed under Creative

Submission Link: https://biomedres.us/submit-manuscript.php
18. Stasinopoulos D (2017) Eccentric Training of Wrist Extensors is Not Enough in the Management of Lateral Elbow Tendinopathy. An Expert Opinion, Ann Orthop Rheumatol 5(2): 1084.

Assets of Publishing with us
BIOMEDICAL
RESEARCHES

